\title{
A note on the initial value problem of fractional evolution equations
}

\author{
Pengyu Chen ${ }^{1 *}$, Xuping Zhang ${ }^{1,2}$ and Yongxiang $\mathrm{Li}^{1}$
}

\section{"Correspondence:} chpengyu123@163.com

'Department of Mathematics, Northwest Normal University, Lanzhou, 730070, P.R. China Full list of author information is available at the end of the article

\begin{abstract}
In this paper, we discuss the continuous dependence of mild solutions on initial values and orders for the initial value problem of fractional evolution equations in infinite dimensional spaces. The results obtained in this paper improve and extend some related conclusions on this topic. This paper can be considered as a contribution to this emerging field.
\end{abstract}

MSC: $35 R 11 ; 47 J 35$

Keywords: fractional evolution equation; initial value problem; continuous dependence

\section{Introduction}

Fractional differential equations have recently come to be considered to be a very powerful tool to help scientists explore the hidden properties of the dynamics of complex systems in various fields of sciences and engineering. In recent years, fractional differential equations played the key role of a fundamental, efficient, and convenient theoretical framework for more adequate modeling of complex dynamic processes. Indeed, we can find numerous applications in viscoelasticity, electromagnetism, diffusion, control, mechanics, physics, signal processing, chemistry, bioengineering, medicine, and in many other areas. For more details as regards fractional differential equations we refer to the monographs by Miller and Ross [1], Podlubny [2] and Kilbas et al. [3], the papers by Eidelman and Kochubei [4] and Lakshmikantham and Vatsala [5], and the survey by Agarwal et al. [6].

In this paper, we are concerned with the continuous dependence of mild solutions on initial values and orders for the initial value problem (IVP) of the fractional evolution equation

$$
\left\{\begin{array}{l}
{ }_{0}^{\mathrm{C}} D_{t}^{\alpha} u(t)+A u(t)=f(t, u(t)), \quad t \geq 0, \\
u(0)=u_{0}
\end{array}\right.
$$

in Banach space $E$, where ${ }_{0}^{C} D_{t}^{\alpha}$ is the Caputo fractional derivative of order $0<\alpha<1, A$ : $D(A) \subset E \rightarrow E$ is a closed linear operator and $-A$ generates a uniformly bounded $C_{0}$ semigroup $T(t)(t \geq 0)$ in $E, f:[0, \infty) \times E \rightarrow E$ is a continuous nonlinear mapping, $u_{0} \in E$.

In recent years, there has been a significant development in the theory of fractional evolution equations. Due to fractional semilinear evolution equations being abstract formulations for many problems arising in engineering and physics, fractional evolution equations

(c) 2015 Chen et al.; licensee Springer. This article is distributed under the terms of the Creative Commons Attribution 4.0 International License (http://creativecommons.org/licenses/by/4.0/), which permits unrestricted use, distribution, and reproduction in any medium, provided you give appropriate credit to the original author(s) and the source, provide a link to the Creative Commons license, and indicate if changes were made. 
have attracted much attention in recent years; see [7-17] and the references cited therein. In addition, some numerical methods have been used to solve space-time or space fractional evolution equations; see [18-23].

However, we observed that all of the existing articles are only devoted to the study of the existence, uniqueness, and controllability of mild solutions for fractional evolution equations; up to now the continuous dependence of mild solutions on parameters for fractional evolution equations has not been considered in the literature. In order to fill this gap, we are concerned with the continuous dependence of mild solutions on the initial values and orders for IVP (1.1).

\section{Preliminaries}

In this section, we introduce some notations, definitions, and preliminary facts which are used in the sequel.

Let $E$ be a Banach space with the norm $\|\cdot\|$ and let $a>0$ be a constant. We denote by $C([0, a], E)$ the Banach space of all continuous $E$-value functions on interval $[0, a]$ with the supnorm $\|u\|_{C}=\sup _{t \in[0, a]}\|u(t)\|$. Throughout this paper, we assume that $A: D(A) \subset E \rightarrow$ $E$ is a closed linear operator and $-A$ generates a uniformly bounded $C_{0}$-semigroup $T(t)$ $(t \geq 0)$ on $E$. Let $M=\sup _{t \in[0,+\infty)}\|T(t)\|_{\mathcal{L}(E)}$, where $\mathcal{L}(E)$ stands for the Banach space of all linear and bounded operators in $E$. For more details of the theory of operator semigroups, see [24].

Let $0<\alpha<1$. The Caputo fractional order derivative of order $\alpha$ with the lower limit 0 for a function $u \in C^{1}[0, a]$ is defined as

$$
{ }_{0}^{C} D_{t}^{\alpha} u(t)=J_{t}^{1-\alpha} u^{\prime}(t)=\frac{1}{\Gamma(1-\alpha)} \int_{0}^{t}(t-s)^{-\alpha} u^{\prime}(s) d s, \quad t>0,
$$

where $\Gamma$ stands for the gamma function, and the fractional integral of order $\alpha>0$ with the lower limit 0 for a function $u$ is defined as

$$
J_{t}^{\alpha} u(t)=\frac{1}{\Gamma(\alpha)} \int_{0}^{t}(t-s)^{\alpha-1} u(s) d s
$$

If $u$ is an abstract function with values in $E$, then the integrals which appear in (2.1) and (2.2) are taken in Bochner's sense. For the details as regards the definitions of fractional derivative and integral, please see [1-3].

Definition 2.1 By a mild solution of IVP $(1.1)$ on $[0, \infty)$, we mean a continuous function $u$ defined from $[0, \infty)$ into $E$ satisfying

$$
u(t)=\mathcal{T}_{\alpha}(t) u_{0}+\int_{0}^{t}(t-s)^{\alpha-1} \mathcal{S}_{\alpha}(t-s) f(s, u(s)) d s, \quad t \in[0, \infty)
$$

where

$$
\begin{aligned}
& \mathcal{T}_{\alpha}(t)=\int_{0}^{\infty} h_{\alpha}(s) T\left(t^{\alpha} s\right) d s, \quad \mathcal{S}_{\alpha}(t)=\alpha \int_{0}^{\infty} s h_{\alpha}(s) T\left(t^{\alpha} s\right) d s, \\
& h_{\alpha}(s)=\frac{1}{\pi \alpha} \sum_{n=1}^{\infty}(-s)^{n-1} \frac{\Gamma(n \alpha+1)}{n !} \sin (n \pi \alpha), \quad s \in(0, \infty),
\end{aligned}
$$


is a function of Wright type defined on $(0, \infty)$ which satisfies

$$
h_{\alpha}(s) \geq 0, \quad s \in(0, \infty), \quad \int_{0}^{\infty} h_{\alpha}(s) d s=1
$$

and

$$
\int_{0}^{\infty} s^{v} h_{\alpha}(s) d s=\frac{\Gamma(1+v)}{\Gamma(1+\alpha v)}, \quad v \in[0,1] .
$$

The following lemma will be of fundamental importance in what follows (see [7, 9]).

Lemma 2.2 The operators $\mathcal{T}_{q}(t)(t \geq 0)$ and $\mathcal{S}_{\alpha}(t)(t \geq 0)$ have the following properties:

(1) For every fixed $t \geq 0, \mathcal{T}_{\alpha}(t)$ and $\mathcal{S}_{\alpha}(t)$ are linear bounded operators, i.e.,

$$
\left\|\mathcal{T}_{\alpha}(t) u\right\| \leq M\|u\|, \quad\left\|\mathcal{S}_{\alpha}(t) u\right\| \leq \frac{M}{\Gamma(\alpha)}\|u\|, \quad u \in E
$$

(2) The operators $\mathcal{T}_{\alpha}(t)(t \geq 0)$ and $\mathcal{S}_{\alpha}(t)(t \geq 0)$ are strongly continuous on $[0, \infty)$.

In what follows, we recall the following Gronwall-Bellman type inequalities, which can be used in fractional differential equations and integral equations with singular kernel.

Lemma 2.3 ([25]) Suppose that $m>0, n \geq 0$, and $q>0$. If $u(t)$ is nonnegative and locally integrable on $0 \leq t<C$ (some $C \leq+\infty)$ with

$$
u(t) \leq m+n \int_{0}^{t}(t-s)^{q-1} u(s) d s
$$

on this interval. Then

$$
u(t) \leq m E_{q}\left(n \Gamma(q) t^{q}\right), \quad 0 \leq t<C,
$$

where $E_{q}$ is the Mittag-Leffler function defined by

$$
E_{q}(z)=\sum_{n=0}^{\infty} \frac{z^{n}}{\Gamma(n q+1)}, \quad z \in \mathbb{C} .
$$

Lemma 2.4 ([26]) Suppose $q>0, m(t)$ is a nonnegative function locally integrable on $0 \leq$ $t<C$ and $n(t)$ is a nonnegative, nondecreasing, continuous function defined on $0 \leq t<C$, $n(t) \leq K$ (constant), and suppose $u(t)$ is nonnegative and locally integrable on $0 \leq t<C$ with

$$
u(t) \leq m(t)+n(t) \int_{0}^{t}(t-s)^{q-1} u(s) d s
$$

on this interval. Then

$$
u(t) \leq m(t)+\int_{0}^{t}\left[\sum_{n=1}^{\infty} \frac{(n(t) \Gamma(q))^{n}}{\Gamma(n q)}(t-s)^{n q-1} m(s)\right] d s, \quad 0 \leq t<C .
$$




\section{Main results}

In this section, we state and prove the main results of this paper. We suppose that the nonlinear term $f$ satisfies the following local Lipschitz condition:

$\left(\mathrm{H}_{f}\right)$ For any $\bar{t}>0$ and constant $r>0$, there exists a positive constant $L=L(r, \bar{t})$ such that

$$
\|f(t, u(t))-f(t, v(t))\| \leq L\|u(t)-v(t)\|
$$

for every $t \in[0, \bar{t}]$ and all $u, v \in E$ satisfying $\|u(t)\| \leq r,\|v(t)\| \leq r$.

Theorem 3.1 Assume that the condition $\left(\mathrm{H}_{f}\right)$ is satisfied, then there exists a constant a $>0$ such that IVP (1.1) has a unique mild solution $u$ on $[0, a]$. Moreover, for any $\epsilon>0$, there exists a constant $\delta>0$ such that for arbitrary $\bar{u}_{0} \in E$ satisfying $\left\|\bar{u}_{0}-u_{0}\right\|<\delta$, the initial value problem (IVP) of the fractional evolution equation

$$
\left\{\begin{array}{l}
{ }_{0}^{\mathrm{C}} D_{t}^{\alpha} u(t)+A u(t)=f(t, u(t)), \quad t \geq 0 \\
u(0)=\bar{u}_{0}
\end{array}\right.
$$

has a unique mild solution $\bar{u}$ on $[0, a]$ and $\|\bar{u}(t)-u(t)\|<\epsilon$ for every $t \in[0, a]$.

Proof We first prove the local existence and uniqueness of mild solutions for IVP (1.1) on interval $\left[0, a_{1}\right]$ for an appropriately small constant $a_{1}>0$, which will be given later. Consider the operator $\mathcal{F}: C\left(\left[0, a_{1}\right], E\right) \rightarrow C\left(\left[0, a_{1}\right], E\right)$ defined by

$$
(\mathcal{F} u)(t)=\mathcal{T}_{\alpha}(t) u_{0}+\int_{0}^{t}(t-s)^{\alpha-1} \mathcal{S}_{\alpha}(t-s) f(s, u(s)) d s, \quad t \in\left[0, a_{1}\right]
$$

From the continuity of nonlinear term $f$ and Lemma 2.2(2) one can easily see that the operator $\mathcal{F}: C\left(\left[0, a_{1}\right], E\right) \rightarrow C\left(\left[0, a_{1}\right], E\right)$ is continuous. By Definition 2.1, the mild solution of IVP (1.1) on $\left[0, a_{1}\right]$ is equivalent to the fixed point of the operator $\mathcal{F}$ defined by (3.2). Let $R=2 M\left\|u_{0}\right\|, L=L(R, 1), N=\max _{0 \leq t \leq 1}\|f(t, \theta)\|$. Denote $\Omega_{R}=\left\{u \in C\left(\left[0, a_{1}\right], E\right):\|u(t)\| \leq\right.$ $\left.R, t \in\left[0, a_{1}\right]\right\}$, then $\Omega_{R}$ is a closed ball in $C\left(\left[0, a_{1}\right], E\right)$ with center $\theta$ and radius $R$. Set

$$
a_{1}:=\min \left\{1,\left(\frac{\Gamma(\alpha+1)\left\|u_{0}\right\|}{L R+N}\right)^{\frac{1}{\alpha}},\left(\frac{\Gamma(\alpha+1)}{M L+1}\right)^{\frac{1}{\alpha}}\right\} .
$$

For any $u \in \Omega_{R}$ and $t \in\left[0, a_{1}\right]$, by Lemma 2.2(1), (3.2), (3.3), and the condition $\left(\mathrm{H}_{f}\right)$, we have

$$
\begin{aligned}
\|(\mathcal{F} u)(t)\| & \leq M\left\|u_{0}\right\|+\frac{M}{\Gamma(\alpha)} \int_{0}^{t}(t-s)^{\alpha-1}(\|f(s, u(s))-f(s, \theta)\|+\|f(s, \theta)\|) d s \\
& \leq M\left\|u_{0}\right\|+\frac{(L R+N) M a_{1}^{\alpha}}{\Gamma(\alpha+1)} \\
& \leq 2 M\left\|u_{0}\right\| \\
& =R .
\end{aligned}
$$

Therefore, $\mathcal{F} u \in \Omega_{R}$. Thus, we proved that $\mathcal{F}: \Omega_{R} \rightarrow \Omega_{R}$ is a continuous operator. Now we show that the operator $\mathcal{F}$ is a contraction in $\Omega_{R}$. For any $u, v \in C\left(\left[0, a_{1}\right], E\right)$, and $t \in\left[0, a_{1}\right]$, 
it follows from Lemma 2.2(1), (3.2), (3.3), and the condition $\left(\mathrm{H}_{f}\right)$ that

$$
\begin{aligned}
\|(\mathcal{F} u)(t)-(\mathcal{F} v)(t)\| & \leq \frac{M}{\Gamma(\alpha)} \int_{0}^{t}(t-s)^{\alpha-1}\|f(s, u(s))-f(s, v(s))\| d s \\
& \leq \frac{M L a_{1}^{\alpha}}{\Gamma(\alpha+1)}\|u-v\|_{C} \\
& <\|u-v\|_{C} .
\end{aligned}
$$

Thus, by (3.5) we have

$$
\|\mathcal{F} u-\mathcal{F} v\|_{C}<\|u-v\|_{C}
$$

which means that $\mathcal{F}$ has a unique fixed point $u \in \Omega_{R}$, which is in turn a unique mild solution of IVP (1.1) on [0, $\left.a_{1}\right]$. Using a similar method we can prove that IVP (3.1) has a unique mild solution $\bar{u}$ on $\left[0, a_{2}\right]$ for appropriate constant $a_{2}>0$. Let $a=\min \left\{a_{1}, a_{2}\right\}$. Then for any $t \in[0, a], u(t)$ and $\bar{u}(t)$ are the unique mild solutions of IVP (1.1) and IVP (3.1), respectively, and they are given by

$$
u(t)=\mathcal{T}_{\alpha}(t) u_{0}+\int_{0}^{t}(t-s)^{\alpha-1} \mathcal{S}_{\alpha}(t-s) f(s, u(s)) d s
$$

and

$$
\bar{u}(t)=\mathcal{T}_{\alpha}(t) \bar{u}_{0}+\int_{0}^{t}(t-s)^{\alpha-1} \mathcal{S}_{\alpha}(t-s) f(s, \bar{u}(s)) d s .
$$

Next, we prove the continuous dependence of the mild solutions on the initial values. For any $\epsilon>0$, choosing $\delta=\frac{\Gamma(\alpha) \epsilon}{M E_{\alpha}\left(M L(R, a) a^{\alpha}\right)+1}$ such that $\left\|u_{0}-\bar{u}_{0}\right\| \leq \delta$, where $E_{\alpha}$ is the MittagLeffler function defined by (2.7). From (3.6), (3.7), Lemma 2.2(1), and the condition $\left(\mathrm{H}_{f}\right)$, we get

$$
\begin{aligned}
\|u(t)-\bar{u}(t)\| & \leq \frac{M}{\Gamma(\alpha)}\left\|u_{0}-\bar{u}_{0}\right\|+\frac{M}{\Gamma(\alpha)} \int_{0}^{t}(t-s)^{\alpha-1}\|f(s, u(s))-f(s, \bar{u}(s))\| d s \\
& \leq \frac{M \delta}{\Gamma(\alpha)}+\frac{M L(R, a)}{\Gamma(\alpha)} \int_{0}^{t}(t-s)^{\alpha-1}\|u(s)-\bar{u}(s)\| d s
\end{aligned}
$$

for any $t \in[0, a]$. Therefore, by (3.8) and Lemma 2.3 one gets

$$
\|u(t)-\bar{u}(t)\| \leq \frac{M \delta}{\Gamma(\alpha)} E_{\alpha}\left(M L(R, a) a^{\alpha}\right)<\epsilon .
$$

This completes the proof of Theorem 3.1.

Next, we discuss the continuous dependence of the mild solutions for IVP (1.1) on the orders.

Theorem 3.2 Assume that the condition $\left(\mathrm{H}_{f}\right)$ is satisfied, then there exists a constant $a>0$ such that for any $0<\beta<1$, for IVP (1.1), and for the IVP of the fractional evolution equation

$$
\left\{\begin{array}{l}
{ }_{0}^{C} D_{t}^{\beta} u(t)+A u(t)=f(t, u(t)), \quad t \geq 0 \\
u(0)=u_{0}
\end{array}\right.
$$


there exist unique mild solutions $u$ and $\tilde{u}$ on $[0, a]$, respectively, and $\|\tilde{u}(t)-u(t)\| \rightarrow 0$ as $\alpha \rightarrow \beta$ for every $t \in[0, a]$.

Proof By using a similar method to the proof of Theorem 3.1, we can prove that for IVP (1.1) and IVP (3.9) there exist unique mild solutions $u$ and $\tilde{u}$ on $[0, a]$ for an appropriate constant $a>0$, respectively, and $u$ and $\tilde{u}$ are given by (3.6) and the following integral equation:

$$
\tilde{u}(t)=\mathcal{T}_{\beta}(t) u_{0}+\int_{0}^{t}(t-s)^{\beta-1} \mathcal{S}_{\beta}(t-s) f(s, \tilde{u}(s)) d s .
$$

Without loss of generality, we may assume that $\alpha>\beta$. Then by (2.4), (3.6), (3.10), Lemma 2.2(1), and the condition $\left(\mathrm{H}_{f}\right)$ one has

$$
\begin{aligned}
\|u(t)-\tilde{u}(t)\| \leq & \left\|\mathcal{T}_{\alpha}(t) u_{0}-\mathcal{T}_{\beta}(t) u_{0}\right\|+\| \int_{0}^{t}(t-s)^{\alpha-1} \mathcal{S}_{\alpha}(t-s) f(s, u(s)) d s \\
& -\int_{0}^{t}(t-s)^{\beta-1} \mathcal{S}_{\beta}(t-s) f(s, \tilde{u}(s)) d s \| \\
\leq & \int_{0}^{\infty}\left\|h_{\alpha}(\tau) T\left(t^{\alpha} \tau\right) u_{0}-h_{\beta}(\tau) T\left(t^{\beta} \tau\right) u_{0}\right\| d \tau \\
& +\frac{M F}{\alpha \Gamma(\beta)} t^{\alpha}-\frac{M F}{\Gamma(\beta+1)} t^{\beta} \\
& +F \int_{0}^{t}(t-s)^{\alpha-1} \int_{0}^{\infty} \tau \| \alpha h_{\alpha}(\tau) T\left((t-s)^{\alpha} \tau\right) \\
& -\beta h_{\beta}(\tau) T\left((t-s)^{\beta} \tau\right) \| d \tau d s \\
& +\frac{M L(R, a)}{\Gamma(\beta)} \int_{0}^{t}(t-s)^{\alpha-1}\|u(s)-\tilde{u}(s)\| d s
\end{aligned}
$$

for every $t \in[0, a]$, where $F=\max _{0 \leq t \leq a}\{\|f(t, u(t))\|:\|u(t)\| \leq R\}$. By (3.11), the properties of the Wright type functions $h_{\alpha}(\cdot), h_{\beta}(\cdot)$, and the semigroup $T(t)(t \geq 0)$, we know that for any $t \in[0, a]$,

$$
\begin{aligned}
m(t):= & \int_{0}^{\infty}\left\|h_{\alpha}(\tau) T\left(t^{\alpha} \tau\right) u_{0}-h_{\beta}(\tau) T\left(t^{\beta} \tau\right) u_{0}\right\| d \tau+\frac{M F}{\alpha \Gamma(\beta)} t^{\alpha}-\frac{M F}{\Gamma(\beta+1)} t^{\beta} \\
& +F \int_{0}^{t}(t-s)^{\alpha-1} \int_{0}^{\infty} \tau \| \alpha h_{\alpha}(\tau) T\left((t-s)^{\alpha} \tau\right) \\
& -\beta h_{\beta}(\tau) T\left((t-s)^{\beta} \tau\right) \| d \tau d s \\
\rightarrow & 0 \quad \text { as } \alpha \rightarrow \beta .
\end{aligned}
$$

Therefore, by (3.11), (3.12), and Lemma 2.4, we get

$$
\begin{aligned}
\|u(t)-\tilde{u}(t)\| & \leq m(t)+\int_{0}^{t}\left[\sum_{n=1}^{\infty} \frac{(M L(R, a) \Gamma(\alpha))^{n}}{\Gamma(\beta)^{n} \Gamma(n \alpha)}(t-s)^{n \alpha-1} m(s)\right] d s \\
& \rightarrow 0 \quad \text { as } \alpha \rightarrow \beta
\end{aligned}
$$

for every $t \in[0, a]$. This completes the proof of Theorem 3.2. 
Remark 3.3 The results obtained in this paper can be applied to all kinds of timefractional partial differential equations. Using Theorems 3.1 and 3.2 one can obtain the continuous dependence results for the concrete fractional partial differential equations. Based on Theorem 3.2, obtained in this paper, we can approximate the solutions of timefractional differential equations by the solutions of integer order differential equations, which are easier to obtain. Therefore, Theorem 3.2 gives an approach to obtain an approximate solution of fractional differential equations.

\section{Competing interests}

The authors declare that they have no competing interests.

\section{Authors' contributions}

All authors contributed equally to the writing of this paper. All authors read and approved the final manuscript.

\section{Author details}

1 Department of Mathematics, Northwest Normal University, Lanzhou, 730070, P.R. China. ${ }^{2}$ Department of Mathematics, Zhixing College of Northwest Normal University, Lanzhou, 730070, P.R. China.

\section{Acknowledgements}

The authors are very grateful to the anonymous referees for their valuable suggestions. This work is supported by NWNU-LKQN-14-3 and NNSF of China (11261053).

Received: 16 November 2014 Accepted: 13 April 2015 Published online: 14 May 2015

\section{References}

1. Miller, KS, Ross, B: An Introduction to the Fractional Calculus and Differential Equations. Wiley, New York (1993)

2. Podlubny, I: Fractional Differential Equations. Academic Press, San Diego (1999)

3. Kilbas, AA, Srivastava, HM, Trujillo, JJ: Theory and Applications of Fractional Differential Equations. North-Holland Mathematics Studies, vol. 204. Elsevier, Amsterdam (2006)

4. Eidelman, SD, Kochubei, AN: Cauchy problem for fractional diffusion equations. J. Differ. Equ. 199, $211-255$ (2004)

5. Lakshmikantham, V, Vatsala, AS: Basic theory of fractional differential equations. Nonlinear Anal. 69, 1677-1682 (2008)

6. Agarwal, RP, Benchohra, M, Hamani, S: A survey on existence results for boundary value problems of nonlinear fractional differential equations and inclusions. Acta Appl. Math. 109, 973-1033 (2010)

7. El-Borai, MM: Some probability densities and fundamental solutions of fractional evolution equations. Chaos Solitons Fractals 14, 433-440 (2002)

8. El-Borai, MM, El-Nadi, KS, El-Akabawy, EG: On some fractional evolution equations. Comput. Math. Appl. 59. 1352-1355 (2010)

9. Wang, J, Zhou, Y: A class of fractional evolution equations and optimal controls. Nonlinear Anal., Real World Appl. 12, 262-272 (2011)

10. Wang, J, Zhou, Y, Fečkan, M: Abstract Cauchy problem for fractional differential equations. Nonlinear Dyn. 74, 685-700 (2013)

11. Wang, R, Xiao, TJ, Liang, J: A note on the fractional Cauchy problems with nonlocal conditions. Appl. Math. Lett. 24, 1435-1442 (2011)

12. Chen, $\mathrm{P}, \mathrm{Li}, \mathrm{Y}$ : Existence of mild solutions for fractional evolution equations with mixed monotone nonlocal conditions. Z. Angew. Math. Phys. 65, 711-728 (2014)

13. Chen, $P, L i, Y, C h e n, Q$, Feng, B: On the initial value problem of fractional evolution equations with noncompact semigroup. Comput. Math. Appl. 67, 1108-1115 (2014)

14. Chen, $P, L i, Y, L i, Q$ : Existence of mild solutions for fractional evolution equations with non-local initial conditions. Ann. Pol. Math. 110, 13-24 (2014)

15. Herzallah, MAE, Baleanu, D: On fractional order hybrid differential equations. Abstr. Appl. Anal. 2014, Article ID 389386 (2014)

16. Herzallah, MAE, El-Shahed, M, Baleanu, D: Mild and strong solutions for a fractional nonlinear Neumann boundary value problem. J. Comput. Anal. Appl. 15, 341-352 (2013)

17. Kerboua, M, Debbouche, A, Baleanu, D: Approximate controllability of Sobolev type nonlocal fractional stochastic dynamic systems in Hilbert spaces. Abstr. Appl. Anal. 2013, Article ID 262191 (2013)

18. Bhrawy, AH, Zaky, MA, Baleanu, D: New numerical approximations for space-time fractional Burgers' equations via a Legendre spectral-collocation method. Rom. Rep. Phys. 67(2), 1-13 (2015)

19. Bhrawy, AH, Baleanu, D: A spectral Legendre-Gauss-Lobatto collocation method for a space-fractional advection diffusion equations with variable coefficients. Rep. Math. Phys. 72, 219-233 (2013)

20. Bhrawy, AH, Al-Zahrani, AA, Alhame, YA, Baleanu, D: A new generalized Laguerre-Gauss collocation scheme for numerical solution of generalized fractional pantograph equations. Rom. J. Phys. 59, 646-657 (2014)

21. Bhrawy, AH, Zaky, MK: A method based on the Jacobi tau approximation for solving multi-term time-space fractional partial differential equations. J. Comput. Phys. 281, 876-895 (2015)

22. Bhrawy, AH, Doha, EH, Baleanu, D, Ezz-Eldien, SS: A spectral tau algorithm based on Jacobi operational matrix for numerical solution of time fractional diffusion-wave equations. J. Comput. Phys. (2014). doi:10.1016/j.jcp.2014.03.039

23. Abdelkawy, MA, Zaky, MA, Bhrawy, AH, Baleanu, D: Numerical simulation of time variable fractional order mobile-immobile advection-dispersion model. Rom. Rep. Phys. 67(3), 1-19 (2015) 
24. Pazy, A: Semigroups of Linear Operators and Applications to Partial Differential Equations. Springer, Berlin (1983)

25. Henry, D: Geometric Theory of Semilinear Parabolic Equations. Lecture Notes in Math., vol. 840. Springer, New York (1981)

26. Ye, $\mathrm{H}, \mathrm{Gao}, \mathrm{J}$, Ding, Y: A generalized Gronwall inequality and its application to a fractional differential equation. J. Math. Anal. Appl. 328, 1075-1081 (2007)

Submit your manuscript to a SpringerOpen ${ }^{\circ}$ journal and benefit from:

- Convenient online submission

Rigorous peer review

- Immediate publication on acceptance

- Open access: articles freely available online

- High visibility within the field

- Retaining the copyright to your article 